\title{
Analysis of Dynamic Spectrum Access with Heterogeneous Networks: Benefits of Channel Packing Scheme
}

\author{
Ling Luo and Sumit Roy \\ Dept. of Electrical Engineering \\ University of Washington \\ Seattle, WA 98195 \\ Email: \{luol,sroy\}@u.washington.edu
}

\begin{abstract}
Dynamic Spectrum Access (DSA) has the potential to vastly improve spectrum utilizations among heterogeneous networks. We present a continuous-time Markov chain (CT-MC) model to analyze the performance of three co-located cognitive systems with various priority classes and bandwidth requirements. The maximum spectrum utilization and minimum blocking probability are derived in a one-channel band scenario. A channel packing scheme (CPS) is then proposed in a multiplechannel band scenario. This scheme packs users of smaller bandwidth requirements in clusters, in order to alleviate the unnecessary blockage to users of larger bandwidth requirements. Numerical results show that the system can benefit from CPS in terms of blocking probability, spectrum utilization and overall failure probability.
\end{abstract}

\section{INTRODUCTION}

Recent studies on wireless spectrum usage have discovered the underutilization of precious spectral resources. To improve the efficiency of spectrum occupancy, the Federal Communication Committee (FCC) has suggested a new policy for dynamic spectrum access (DSA) [1]. The concept of cognitive radio (CR) [2] on top of DSA is introduced as a promising technique to alleviate the scarcity of spectral resources. CR network specifies a certain method for unlicensed (secondary) users to access the spectrum without causing harmful interference to authorized (primary) users. The major challenges in latest research of $\mathrm{CR}$ networks include: (a) analysis of DSA with homogeneous/heterogeneous networks and (b) reliable and effective channel sensing schemes. Both these two aspects are explored in this paper.

Current research of DSA channel model analysis mainly focuses on homogeneous networks. Chou [3] provides the basic M/G/1 model for the CR network.
Further analysis is given by Tang [4] and Zhu [5] in terms of blocking probability and queueing delay. Their research is based on the assumption that primary and secondary systems have the same bandwidth requirements. Although the practical cases of DSA might include heterogeneous networks of different bandwidth requirements, the reference regarding these considerations is still limited. Yiping [6] gives a continuoustime Markov chain (CT-MC) model for two types of secondary systems, but the analysis does not include primary systems. Zhu [7] presents the CT-MC model for one primary system and one secondary system while the case is still simple and the channel reservation scheme does not satisfy the agile property of cognitive users. Raspopovic [8] presents a similar model but not for DSA.

In this paper, we set up a CT-MC model for DSA with heterogeneous networks. We assume multiple radio systems of diverse bandwidth requirements attempting to operate in the same band. This paper provides the channel modeling for spectrum sharing of three different systems: one is primary and the other two are secondary. One secondary system has the same bandwidth requirement as the primary system while the other does not. The analysis of CT-MC model for heterogeneous networks is provided and the optimal spectrum utilization and blocking probability for DSA with three systems are derived in a one-channel band scenario.

Other than modeling DSA, this paper also presents a cross-layer channel occupation (sensing) scheme. Upto-date research about spectrum sensing in DSA mainly focuses on approaches on PHY or MAC layer. Some optimal sensing schemes in terms of reducing sensing overhead and increasing cooperation are introduced by Kim [9] and Ganesan [10], but they cannot be applied to diverse secondary systems. Zhao [11] provides a 
myopic sensing scheme based on analysis of the discretetime Markov channel model while the heterogeneous networks are not considered. Liang [12] investigates the optimal sensing time to maximize the throughput, where cooperative sensing is still hard to implement in DSA because of the opaque information exchange of different systems. As an easy implementation to MAC and PHY layer, the cross-layer approach of channel sensing can coordinate different requirements of heterogeneous networks. Su [13] gives a cross-layer MAC protocol and the queueing model analysis. The conventional occupation schemes (random search and serial search) are usually considered in cross-layer design. It is shown in Section III that these conventional schemes lead to the unnecessary blockage of secondary users. Based on an idea of two-stage sensing scheme given by Ling [14], we then present a channel packing scheme which can reach the upper bound of the number of secondary users that can operate in DSA with heterogeneous networks. We evaluate the performance in a multiple-channel band scenario. It is shown that the system benefits from our scheme in terms of blocking probability, spectrum utilization and overall failure probability.

\section{DSA FOR HETEROGENEOUS SYSTEMS: FORMULATION}

We assume that multiple radio systems simultaneously operate in one band. There is a master node which controls the arrival rates for two secondary systems. The primary system is considered to hold the highest priority while other secondary systems have the same lower class. The representative rules of occupations for these different types of users are defined as below:

Rule 1: An arrival of a primary user wishing to access a channel currently occupied by any secondary user will cause the secondary user to vacate that channel.

Rule 2: The secondary user that vacates a channel due to the arrival of a primary user will occupy another available channel in a very short transition time $T_{t s}$, if the channel satisfies the bandwidth requirement of this secondary user.

Rule 3: An arrival of a primary user wishing to access a channel currently occupied by another primary user will cause blockage of the new primary user.

Rule 4: An arrival of a secondary user wishing to access a channel currently occupied by another secondary/primary user will cause blockage of the new secondary user.

In this paper, we consider the case of three colocated systems- a primary system $(A)$ and two secondary

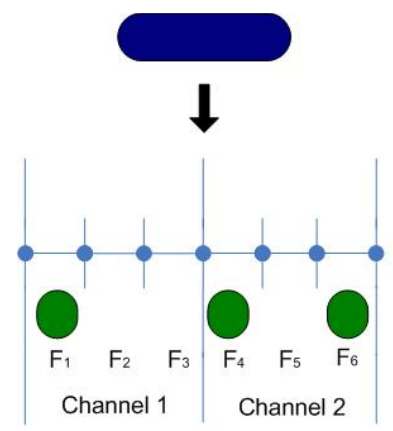

(a). System C: Random Search Type C Use

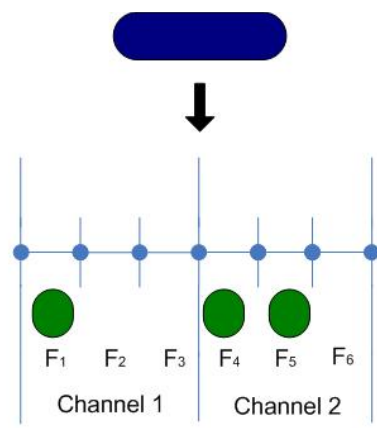

(b). System C: Serial Search
Type B User

Fig. 1. Blockage of a secondary user of large bandwidth

systems $(B, C)$, where the bandwidth requirement for each user in these three systems follows ( $k$ is an integer)

$$
B_{A}=B_{B}=k B_{c}, k>1
$$

where a type $A$ user has the same bandwidth as a type $B$ user, and $k$ times a type $C$ user $\left(B_{i}\right.$ is the bandwidth that a type $i$ user will occupy per time).

The system band of interest equals $N$ channels of type $A / B$ users, and thus

$$
B_{\text {overall }}=N B_{A}=N B_{B}=N k B_{C}
$$

where $B_{\text {overall }}$ is the bandwidth of the whole band.

We assume no buffer for queueing of both primary and secondary systems. Thus any user blocked by the crowded spectrum or terminated by primary users will just leave the band, if there is no available channel. We also assume perfect signal detection and model the offered traffic with three Poisson random processes. Each system $i$ has an arrival rate $\lambda_{i}$, and a the probability distribution of its service time is exponential with mean $1 / \mu_{i}$. The CT-MC analysis of such a three-system case in a one-channel band scenario is given in Section IV, followed by the numerical results in a multiple-channel band scenario in Section V.

\section{Channel Packing Scheme}

For the two secondary systems, an arrival of a type $B$ user might be blocked by the currently active type $C$ users, and vice versa. An effective sensing scheme is then needed to decrease the blocking probability of secondary systems. In the conventional uncooperative schemes, secondary users search for the unoccupied channels either randomly or serially in the band. However, both these schemes lead to the unnecessary blockage of sec- 
ondary systems with large bandwidth requirements. For example, Fig. 1 shows two cases (using random search and serial search) that an arrival of a type $B$ user is blocked, though the overall remaining bandwidth is no smaller than the bandwidth of a type $B$ signal. In both cases, there are two channels and each channel contains three equal-size sub-channels, where a type $B$ user can occupy one channel and a type $C$ user can occupy one sub-channel per time. In Fig.1(a), three type $C$ users randomly locate in the band so that an incoming type $B$ user cannot obtain an available channel. In Fig.1(b), two type $C$ users are occupying two consecutive subchannels, $F_{4}$ and $F_{5}$, since a type $B$ user was using channel 1. After this type $B$ user leaves the band, another type $C$ user comes in and finds that the first sub-channel $\left(F_{1}\right)$ is available via serial search. It then seizes $F_{1}$, leading to the blockage of an incoming type $B$ user thereafter.

Cooperative sensing will be a good approach to address this problem, but it is difficult to be implemented, because most systems are different and they do not share information with each other. We present a novel uncooperative channel packing scheme (CPS) for secondary systems as follows: Suppose there are two types of secondary systems $B$ and $C$, with $B_{B}=k B_{C}$. A type $B$ user apply serial search to locate an available channel while type $C$ users take two steps:

1. Serially search for a channel that some of its subchannels are occupied by type $C$ users while some are free.

2. If there is such a channel, use serial search to occupy an available sub-channel inside it. Otherwise, apply serial search to occupy an available sub-channel in the whole band.

The reason that CPS applies serial search instead of random search includes: a). serial search can pack all type $C$ users in clusters while random search results in dispersion of sub-channel occupations of type $C$ users; b). serial search has the same mean time to detect a free channel as random search [14]. The flowchart diagram of CPS is shown in Fig.2. The complexity of CPS is $O(N k)$, which is the same as that of serial search and random search.

For a band composed of $n$ channels and $m$ type $C$ users, the constraint $m \leq(n-1) k$ means that the aggregate available spectrum resources suffice for the occupation of at least one type $B$ user. Because there is no more than one channel that some of its sub-channels are occupied by type $C$ users while some are free, the largest number of type $B$ users can operate in this band

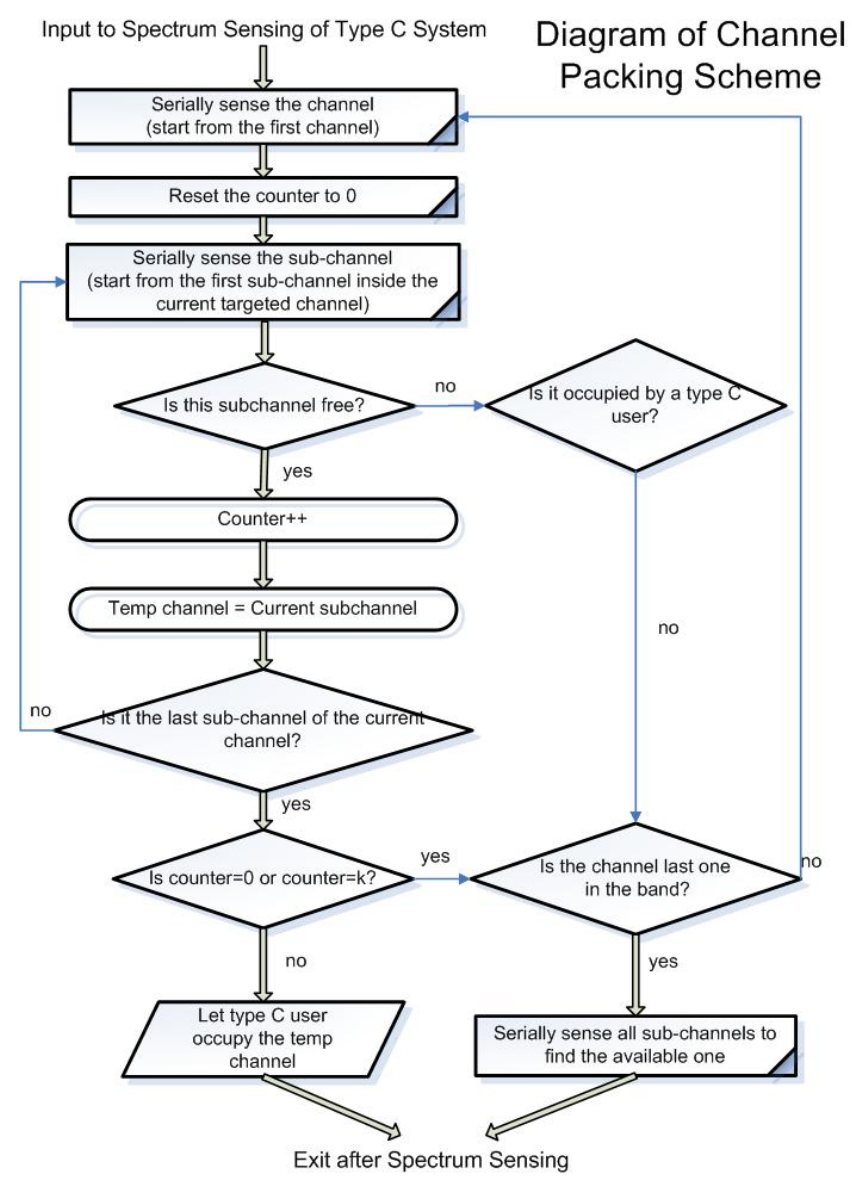

Fig. 2. Flowchart diagram of channel packing scheme

(h) is

$$
h=n-\lceil m / k\rceil
$$

where $\lceil x\rceil$ denotes the smallest integer that $x$ is no larger than. It is the upper bound that such a band can provide via any schemes because

$$
(n-\lceil m / k\rceil+1) k+m>n k
$$

\section{Performance Analysis in One-Channel BAND SCENARIO}

We assume a one-channel band scenario with $k=2$, where the term 'one-channel' means that the whole band has only one channel for the primary system. We assume that the overall bandwidth is $20 \mathrm{MHz}$. System $A$ denotes the primary system with $20 \mathrm{MHz}$ per channel (e.g., IEEE 802.16e signals); system $B$ denotes the secondary system with $20 \mathrm{MHz}$ per channel (e.g., IEEE $802.11 \mathrm{~b}$ signals); system $C$ denotes the secondary system with $10 \mathrm{MHz}$ per channel (e.g., IEEE 802.11p signals). There is just one channel for the primary system in the whole band, and 


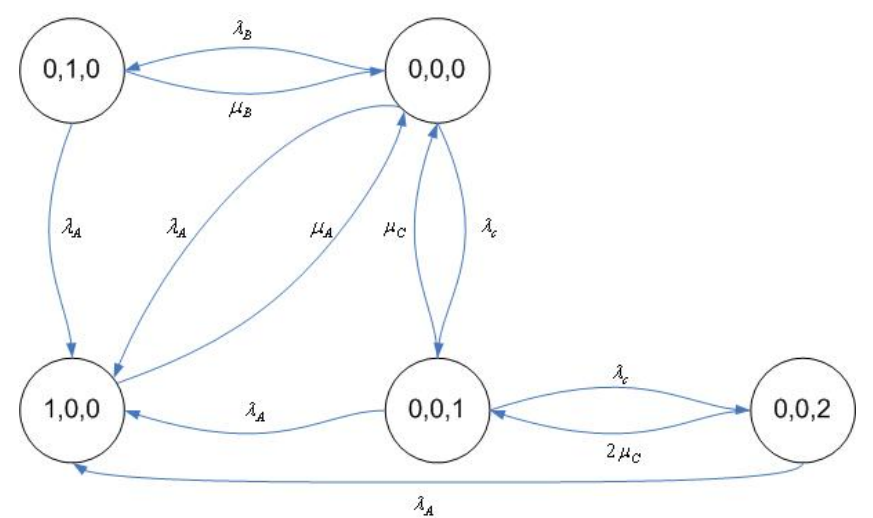

Fig. 3. CT-MC of three-system in one-channel band

the CT-MC is modeled in Fig.3. State $(a, b, c)$ denotes the numbers of type $A / B / C$ users in the current band.

By solving this CT-MC, we can form the relationship among five steady state probabilities $P_{1,0,0}, P_{0,1,0}, P_{0,0,1}$, $P_{0,0,2}$ and $P_{0,0,0}$.

$$
\begin{aligned}
P_{1,0,0} \mu_{A} & =\left(P_{0,0,0}+P_{0,1,0}+P_{0,0,1}+P_{0,0,2}\right) \lambda_{A} \\
P_{0,0,0} \lambda_{B} & =P_{0,1,0}\left(\mu_{B}+\lambda_{A}\right) \\
P_{0,0,0} \lambda_{C} & =P_{0,1,0}\left(\mu_{C}+\lambda_{A}+\lambda_{C}\right)-P_{0,0,2} 2 \mu_{C} \\
P_{0,0,1} \lambda_{C} & =P_{0,0,2}\left(2 \mu_{C}+\lambda_{A}\right) \\
1 & =\sum\left(P_{a, b, c}\right)
\end{aligned}
$$

Thus the values of steady state probabilities are

$$
\begin{aligned}
P_{1,0,0} & =\frac{\lambda_{A}}{\lambda_{A}+\mu_{A}} \\
P_{0,1,0} & =\frac{\lambda_{B}}{\lambda_{A}+\mu_{B}} P_{0,0,0} \\
P_{0,0,1} & =H \cdot P_{0,0,0} \\
P_{0,0,2} & =H \frac{\lambda_{C}}{2 \mu_{C}+\lambda_{A}} P_{0,0,0} \\
P_{0,0,0} & =\frac{\mu_{A} /\left(\lambda_{A}+\mu_{A}\right)}{1+\frac{\lambda_{B}}{\lambda_{A}+\mu_{B}}+H\left(\frac{\lambda_{C}}{2 \mu_{C}+\lambda_{A}}+1\right)}
\end{aligned}
$$

where $H=\frac{\lambda_{C}}{\left(\lambda_{A}+\mu_{C}+\lambda_{C}\right)-\frac{2 \mu_{C} \lambda_{C}}{2 \mu_{C}+\lambda_{A}}}$.

The average spectrum utilization is defined as the fraction of a unit time per unit bandwidth (normalized by $B_{\text {overall }}$ ) occupied by any system. Because we assume equal traffic load, this spectrum utilization then corresponds to the throughput offered by each system. Due to the PASTA property, the average spectrum utilizations of three systems in this scenario are given by

$$
\overline{S U}_{A}=P_{1,0,0}, \overline{S U}_{B}=P_{0,1,0}, \overline{S U}_{C}=P_{0,0,1} / 2+P_{0,0,2}
$$

The lower priority class of secondary users leads to blockage of arrivals if no available channels can be used. On the other hand, an arrival of a primary user will be blocked only if all channels are occupied by primary users. The blocking probability $P_{b}^{i}$ is defined as the probability that an arrival of a type $i$ user is blocked. Thereby, we can derive the blocking probability for each system as below. Even though there is a spectrum hole in the state $(0,0,1)$, the arrival of a type $B$ user will still be blocked because it is not able to utilize the residual spectrum resource.

$$
\begin{aligned}
& P_{A}^{b}=P_{1,0,0} \\
& P_{B}^{b}=P_{1,0,0}+P_{0,1,0}+P_{0,0,1}+P_{0,0,2} \\
& P_{C}^{b}=P_{1,0,0}+P_{0,1,0}+P_{0,0,2}
\end{aligned}
$$

We assume that three systems apply the same pattern of packets and the same transmission rates per bandwidth. Therefore, system $A$ and $B$ have the same processing rates, two times as system $C$. We also assume that the master node applies a gateway over two secondary systems so that the sum of their arrival rates is a constant value $\lambda_{\text {sec }}$. Thus we have

$$
\mu_{A}=\mu_{B}=2 \mu_{C}=\mu, \lambda_{B}+\lambda_{C}=\lambda_{s e c}
$$

The higher spectrum utilization and the lower blocking probability are preferred. The respective spectrum utilization of type $i$ system is increasing as its arrival rate goes up. We will then derive the maximum aggregate spectrum utilization, $\left(\sum \overline{S U}_{i}\right)_{\max }$, as below.

$$
\begin{aligned}
& \qquad \begin{aligned}
\sum \overline{S U}_{i}= & P_{1,0,0}+P_{0,1,0}+P_{0,0,1} / 2+P_{0,0,2} \\
= & 1-P_{0,0,0}-P_{0,0,1} / 2 \\
\left(\sum \overline{S U}_{i}\right)_{\max }= & 1-f_{1}\left(\lambda_{C}\right)_{\min }, 0 \leq \lambda_{C} \leq \lambda_{\text {sec }} \\
\text { where } f_{1}\left(\lambda_{C}\right)_{\text {min }}= & P_{0,0,0}+P_{0,0,1} / 2 .
\end{aligned}
\end{aligned}
$$

By solving $\frac{\partial}{\partial \lambda_{C}} f_{1}\left(\lambda_{C}\right)=0$, we find that there is only one stationary point $\lambda_{C}^{*} \in\left[0, \lambda_{\text {sec }}\right]$. We also notice that $\frac{\partial^{2}}{\partial^{2} \lambda_{C}} f_{1}\left(\lambda_{C}\right)<0$, implying that it is the relative maximal point. The derivations of solving partial derivatives are omitted due to space limitations. Because $f_{1}(0)<f_{1}(\lambda)$, we conclude that the highest aggregate spectrum utilization is achieved when $\lambda_{B}=\lambda_{s e c}, \lambda_{C}=0$. The higher arrival rate of type $C$ system causes more unnecessary blockage of type $B$ users. This result implies that the increasing arrival rate of type $B$ system can better utilize the spectrum resources in terms of aggregate throughput of secondary system.

$$
\left(\sum \overline{S U}_{i}\right)_{\max }=\frac{\lambda_{s e c}+\lambda_{A}}{\mu+\lambda_{s e c}+\lambda_{A}}
$$


For the primary system, the increasing arrival rate will raise the average time of fully occupied state as well as the blocking probability. In this scenario, when the whole band is occupied by a type $A$ user, any incoming user will be blocked. If one sub-channel is occupied by a type $C$ user while the other is available, only an arrival of a type $B$ user will be blocked. Both the cases that the band is fully occupied by type $B$ or $C$ users will result in blockage of incoming type $B$ or $C$ users. Because $P_{1,0,0}$ is a constant value, the blocking probability of primary system $A$ is also constant. Under the constraint that two secondary systems have the constant sum of arrival rates, the decrease in the arrival rate of one secondary system might not lead to the reduction of its blocking probability, due to the augment in the arrival rate of the other secondary system. Our goal is to analyze the minimum blocking probability of both secondary systems $\left(P_{B}^{b}, P_{C}^{b}\right)$ and also the overall blocking probability $\left(P_{o}^{b}\right)$. The latter one is defined as the probability that blockage is occurred conditioning on an arrival of any secondary user.

$$
\begin{aligned}
P_{o}^{b} & =\left(P_{B}^{b} \lambda_{B}+P_{C}^{b} \lambda_{C}\right) /\left(\lambda_{B}+\lambda_{C}\right) \\
\left(P_{B}^{b}\right)_{\min } & =1-f_{2}\left(\lambda_{C}\right)_{\max } \\
\left(P_{C}^{b}\right)_{\min } & =1-f_{3}\left(\lambda_{C}\right)_{\max } \\
\left(P_{o}^{b}\right)_{\min } & =1-f_{4}\left(\lambda_{C}\right)_{\max }
\end{aligned}
$$

where $f_{2}\left(\lambda_{C}\right)=P_{0,0,0}\left(\lambda_{C}\right), f_{3}\left(\lambda_{C}\right)=P_{0,0,0}\left(\lambda_{C}\right)+$ $P_{0,0,1}\left(\lambda_{C}\right)$ and $f_{4}\left(\lambda_{C}\right)=P_{0,0,0}\left(\lambda_{C}\right)+\frac{\lambda_{C}}{\lambda_{s e c}} P_{0,0,1}\left(\lambda_{C}\right)$.

By solving $\frac{\partial}{\partial \lambda_{C}} f_{2}\left(\lambda_{C}\right)=0$ we find that the only stationary point is $\lambda_{C}^{*}=-\mu-\lambda_{A}$, which means that $f_{2}$ is a monotonic function in $\left[0, \lambda_{\text {sec }}\right]$. Because $f_{2}(0)>$ $f_{2}\left(\lambda_{\text {sec }}\right)$, the minimum blocking probability of system $B$ is achieved when $\lambda_{B}=\lambda_{s e c}, \lambda_{C}=0$, and

$$
\left(P_{B}^{b}\right)_{\min }=\frac{\lambda_{s e c}+\lambda_{A}}{\mu+\lambda_{s e c}+\lambda_{A}}
$$

which implies that system $B$ suffers more from the blockage caused by system $C$ than by itself. To minimize the blocking probability, the best choice for system $B$ is to grab all the opportunities to utilize the band and make system $C$ silent.

It is interesting that the minimum blocking probability of type $C$ system is achieved when the arrival rate of type $B$ system is nonzero under some cases. The only possible positive stationary point of $f_{3}$ is

$\lambda_{C}^{\prime *}=\frac{\sqrt{K_{1}^{2} K_{2}^{2} \mu^{2}+K_{1}^{2} K_{2} K_{3} \mu\left(2 K_{1}+K_{2}\right)}-K_{1} K_{2} \mu}{\mu\left(2 K_{1}+K_{2}\right)}$

where $K_{1}=\mu+\lambda_{A}, K_{2}=\mu+2 \lambda_{A}$ and $K_{3}=\mu+$

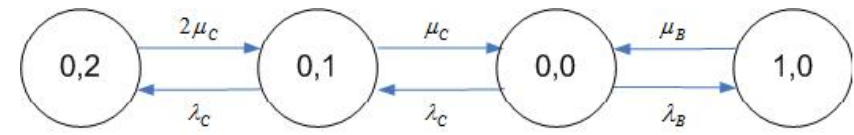

Fig. 4. CT-MC of two-system in one-channel band

$2 \lambda_{A}+2 \lambda_{\text {sec }}$. It satisfies $0 \leq \lambda_{C} \leq \lambda_{\text {sec }}$ only if

$$
\left(2 K_{1}+K_{2}\right) \mu \lambda_{\text {sec }}^{2}+2 K_{1} K_{2} \mu \lambda_{\text {sec }}-K_{1}^{2} K_{2} K_{3} \geq 0
$$

If the condition above holds, the fact $\frac{\partial^{2}}{\partial^{2} \lambda_{C}} f_{3}\left(\lambda_{C}\right)<0$ shows that it is a relative maximal point; otherwise the maximal point is $\lambda_{\text {sec }}$, because $f_{3}(0)<f_{3}\left(\lambda_{\text {sec }}\right)$. When the arrival rate of type $A$ system is low and $\lambda_{\text {sec }}>\mu$, the band is always crowded by secondary users. Keeping a certain value of type $B$ system $\left(\lambda_{B}>0\right)$ leads to the benefit of blocking probability for type $C$ system.

$$
\left(P_{C}^{b}\right)_{\min }=1-f_{3}\left(\min \left(\lambda_{C}^{*}, \lambda_{s e c}\right)\right)
$$

Similarly as $f_{2}$, the only feasible stationary point of $f_{4}$ is $\lambda_{C}^{*}=0$, where $f_{4}(0)<f_{4}\left(\lambda_{\text {sec }}\right)$. The minimum overall blocking probability is achieved when $\lambda_{B}=$ $0, \lambda_{C}=\lambda_{s e c}$.

$$
\left(P_{o}^{b}\right)_{\min }=1-\frac{K_{2} \mu \lambda_{\text {sec }}+K_{1} K_{2} \mu}{2 K_{1} \lambda_{\text {sec }}^{2}+K_{1} K_{2} \lambda_{\text {sec }}+K_{1}^{2} K_{2}}
$$

\section{A. Special Case: No Primary System}

Taking $\lambda_{A}=0(k=2)$ corresponds to the case that no primary system exists and the spectrum is shared by two secondary systems. The CT-MC diagram of this case is shown in Fig.4, where state $(b, c)$ denotes the numbers of type $B / C$ users in the band.

Solving the steady state probabilities in this scenario, we have

$$
\begin{aligned}
P_{0,0} & =1 /\left(1+\frac{\lambda_{C}^{2}}{2 \mu_{C}^{2}}+\frac{\lambda_{C}}{\mu_{C}}+\frac{\lambda_{B}}{\mu_{B}}\right) \\
P_{1,0} & =\frac{\lambda_{B}}{\mu_{B}} /\left(1+\frac{\lambda_{C}^{2}}{2 \mu_{C}^{2}}+\frac{\lambda_{C}}{\mu_{C}}+\frac{\lambda_{B}}{\mu_{B}}\right) \\
P_{0,1} & =\frac{\lambda_{C}}{\mu_{C}} /\left(1+\frac{\lambda_{C}^{2}}{2 \mu_{C}^{2}}+\frac{\lambda_{C}}{\mu_{C}}+\frac{\lambda_{B}}{\mu_{B}}\right) \\
P_{0,2} & =\frac{\lambda_{C}^{2}}{2 \mu_{C}^{2}} /\left(1+\frac{\lambda_{C}^{2}}{2 \mu_{C}^{2}}+\frac{\lambda_{C}}{\mu_{C}}+\frac{\lambda_{B}}{\mu_{B}}\right)
\end{aligned}
$$

By analyzing the similar CT-MC analysis under the constraint (1), we will find that the maximum spectrum utilization of the whole system is $\lambda_{\text {sec }} /\left(\mu+\lambda_{\text {sec }}\right)$, when the master node only allows system $B$ to access the spectrum $\left(\lambda_{B}=\lambda_{s e c}, \lambda_{C}=0\right)$. On the other hand, the 


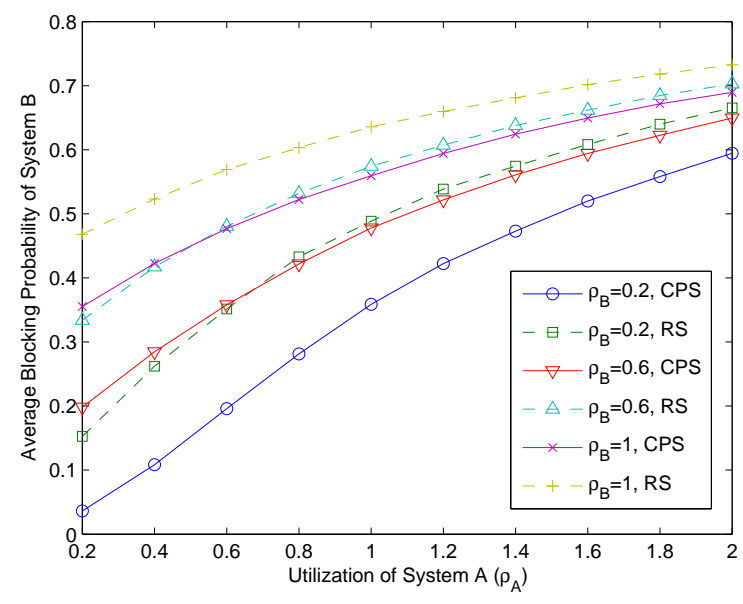

Fig. 5. Average blocking probability of system $B$

minimum overall blocking probability is $\frac{2 \lambda s e c^{2}}{2 \lambda_{\text {sec }}^{2}+\mu \lambda_{s e c}+\mu^{2}}$, when system $B$ keeps silent. It matches the result of (2) and (5).

\section{Numerical Results in Multiple-BAND SCENARIO}

Different sensing schemes perform similarly in the aforementioned one-channel band scenario, but they have variant performance in the multiple-channel scenario, as a result of mutual blockage among secondary systems. It is difficult to describe the CT-MC diagram in a twodimensional plane and obtain the closed form derivation, so we evaluate the performance of conventional random search (RS) and our channel packing scheme (CPS) in numerical results via Matlab. We assume that $\mu_{A}=$ $\mu_{B}=2 \mu_{C}=\mu$, where $\rho_{C}=\lambda_{C} /\left(N k \mu_{C}\right)=0.6$ and $N=5, k=2$. We then tune the utilization factors of system $A$ and $B\left(\rho_{A}=\lambda_{A} /\left(N \mu_{A}\right), \rho_{B}=\lambda_{B} /\left(N \mu_{B}\right)\right)$ to investigate the system issues such as spectrum utilization, blocking probability, forced termination probability and overall failure probability.

When the band is not fully occupied, any incoming cognitive type $C$ user will find an available sub-channel in a short time even though the first try fails, no matter via CPS or RS. If some type $C$ users are forced to terminate by type $A$ users, they can also locate the available sub-channels in a very short transition time. Thus system $C$ obtains the same performance applying CPS as applying RS. We then show the benefits of CPS towards system $B$.

Fig.5 shows that system $B$ obviously benefits from CPS in terms of blocking probability. This benefit keeps

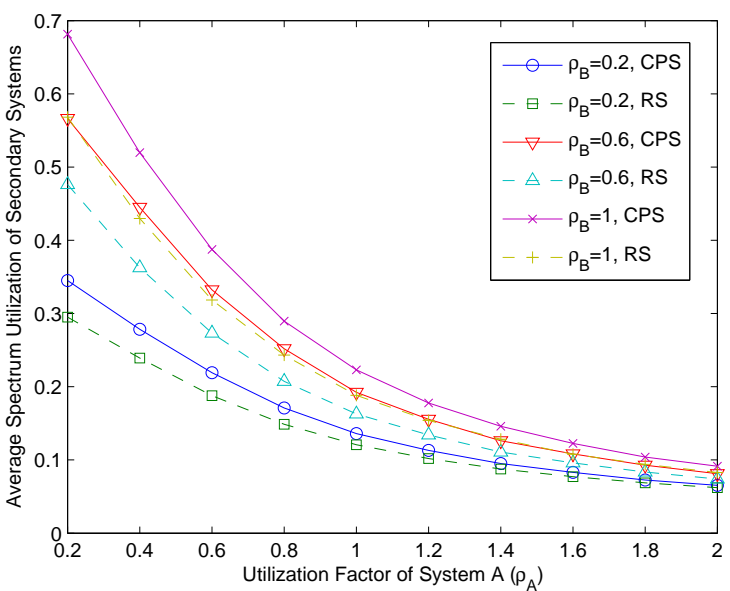

Fig. 6. Average spectrum utilization of system $B$

decreasing as growing of the arrival rate of type $A$ users. The reason is that the increasing number of primary users will decrease the spectrum opportunities for secondary users. The advantage of packing type $C$ users will be not as apparent as in the case of less type $A$ users. It can be also noticed from Fig.6 that applying CPS leads to $10 \%-20 \%$ gain in spectrum utilization of system $B$, especially for $\rho_{A}<1$.

As the less blocking probability causes the longer occupation time of system $B$ in the band, the forced termination probability of system $B$ is also increased. Regarding this trade-off, we take into account a parameter so-called overall failure probability, which is defined as the aggregate probability of failed transmission caused by blockage or forced termination. Fig.7 demonstrates that even though applying CPS results in a little higher forced termination probability of system $B$, the overall failure probability is less than using RS. This result implies that system $B$ obtains more chances to occupy the spectrum via CPS.

\section{CONCLUSION}

A CT-MC model of DSA with heterogeneous networks has been presented in this paper. The analysis in the one-channel band scenario derives the minimum blocking probabilities and maximum spectrum utilizations of three co-located systems with different bandwidth requirements. An uncooperative channel packing scheme is then proposed in the multiple-channel band scenario. Numerical results show that our scheme can modestly decrease the blocking probability and overall failure probability of secondary systems. The secondary 


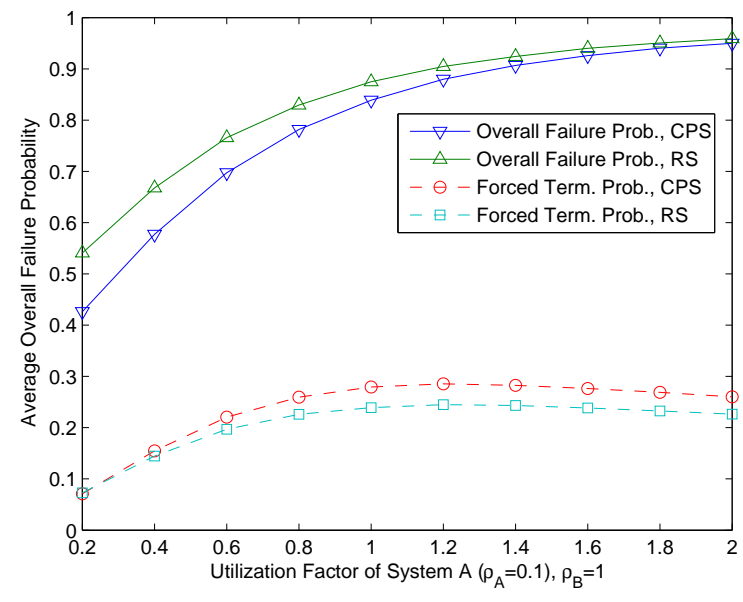

Fig. 7. Avg. forced term. and overall failure prob. of system $B$

systems can also gain $10 \%-20 \%$ in average spectrum utilization when the utilization factor of the primary system is less than 1 .

\section{REFERENCES}

[1] FCC, Spectrum Policy Task Force Report, ET Docket, No.03155, Nov. 2002.

[2] J. Mitola, "Cognitive radio: an integrated agent architecture for software defined radio", Ph.D Thesis, Royal Institute of Techonology (KTH), 2000.

[3] CT. Chou, N.S. Shankar, H. Kim and K.G. Shin, "What and How Much to Gain by Spectrum Agility?", IEEE J. Sel. Areas Commun., Vol. 25, Issue 3, pp. 576-588, Apr. 2007.
[4] S. Tang and B.L. Mark, "Modeling an Opportunistic Spectrum Sharing System with a Correlated Arrival Process", WCNC'08, pp. 3297-3302, Mar. 2008.

[5] P. Zhu, J. Li and X. Wang, "Scheduling Model for Cognitive Radio”, CrownCom'08, pp. 1-6, May 2008.

[6] Y. Xing, R. Chandramouli, S. Mangold and N.S. Shankar, "Dynamic Spectrum Access in Open Spectrum Wireless Networks", IEEE J. Sel. Areas Commun., Vol. 24, Issue 3, pp. 626-637, Mar. 2006.

[7] X. Zhu, L. Shen and T-S.P. Yum, "Analysis of Cognitive Radio Spectrum Access with Optimal Channel Reservation", IEEE Commu. Letters, Vo. 11, Issue 4, pp. 304-306, Apr. 2007.

[8] M. Raspopovic and C. Thompson, "Finite Population Model for Performance Evaluation between Narrowband and Wideband Users in the Shared Radio Spectrum", DySPAN'07, pp. 340346, Apr. 2007.

[9] H. Kim and K.G. Shin, "In-band Spectrum Sensing in Cognitive Radio Networks: Energy Detection of Feature Detection?", ACM MobiCom'08, pp. 14-25, Sep. 2008.

[10] G. Ganesan and Y. Li, "Cooperative Spectrum Sensing in Cognitive Radio Networks”, DySPAN'05, pp.137-143, Nov. 2007.

[11] Q. Zhao, B. Krishnamachari and K. Liu, "On Myopic Sensing for Multi-Channel Opportunistic Access: Structure, Optimality, and Performance", IEEE Trans. on Wireless Commun., Vol. 7, Issue 12, Part 2, pp. 5431-5440, Dec. 2008.

[12] Y-C. Liang, Y. Zeng, E. Peh and A.T. Hoang "SensingThroughput Tradeoff for Cognitive Radio Networks", IEEE Trans. on Wireless Commun., Vol. 7, Issue 4, pp. 1326-1337, Apr. 2008.

[13] H. Su and X. Zhang, "Cross-Layer Based Opportunistic MAC Protocols for QoS Provisionings over Cognitive Radio Wireless Networks", IEEE J. Sel. Areas Commun., Vol. 26, Issue 2, pp. 119-128, Jan. 2008.

[14] L. Luo, N. Neihart, S. Roy and D. Allstot, "A Two-stage Sensing Technique for Dynamic Spectrum Access", to Appear in IEEE Trans. on Wireless Commun. 